Démarches transfrontalières pionnières sur le Rhin supérieur - Une rétrospective des débuts du façonnement d'un paysage européen du savoir

Pioneering cross-border approaches on the Upper Rhine

Die ersten gemeinsamen Forschungsschritte als Grundlage der heutigen europäischen Wissenslandschaft am Oberrhein - Retroperspektive der Pioniere

\title{
Gabriel Wackermann
}

\section{(2) OpenEdition}

Journals

Édition électronique

URL : http://journals.openedition.org/rge/1684

DOI : $10.4000 /$ rge. 1684

ISSN : 2108-6478

Éditeur

Association des géographes de l'Est

Édition imprimée

Date de publication : 1 septembre 2008

ISSN : 0035-3213

Référence électronique

Gabriel Wackermann, « Démarches transfrontalières pionnières sur le Rhin supérieur - Une

rétrospective des débuts du façonnement d'un paysage européen du savoir », Revue Géographique de l'Est [En ligne], vol. 48 / 3-4 | 2008, mis en ligne le 02 mars 2010, consulté le 08 septembre 2020. URL http://journals.openedition.org/rge/1684; DOI : https://doi.org/10.4000/rge.1684

Ce document a été généré automatiquement le 8 septembre 2020.

Tous droits réservés 


\section{Démarches transfrontalières} pionnières sur le Rhin supérieur Une rétrospective des débuts du façonnement d'un paysage européen du savoir

Pioneering cross-border approaches on the Upper Rhine

Die ersten gemeinsamen Forschungsschritte als Grundlage der heutigen europäischen Wissenslandschaft am Oberrhein-Retroperspektive der Pioniere

Gabriel Wackermann

\section{Observations préliminaires et périmètre concerné}

1 Les territoires du savoir, fondés sur des sociétés du savoir, sont le résultat de la mise en œuvre conjointe des talents, du savoir et du savoir-faire, voire du savoir-être, à même de promouvoir une créativité conforme aux exigences des progrès technologiques et de l'aspiration à un mieux-être de plus en plus affiné. Une démarche conforme à l'actuelle compétition mondiale, dans le cadre de la globalisation. Le Rhin supérieur constitue l'une des régions européennes du savoir, une «Wissensregion » originale façonnée à la faveur de la construction européenne. Territoire transfrontalier trinational -franco-germanosuisse- dans sa partie méridionale, prolongé par sa forte empreinte alémanique jusqu’à la hauteur de Francfort $s$ /Main, cet espace naguère fait de pièces et de morceaux est devenu, durant la seconde moitié du XX e siècle, au prix de nombreux efforts visant à surmonter des obstacles tenaces, le creuset de l'une des deux euro-régions emblématiques aux démarches pionnières, toutes les deux de facture rhénane. Appuyée sur la région TriRhena (Schneider-Sliwa, 2003), elle a contribué à structurer le bassin rhénan méridional, comme l'autre euro-région historique, l'Euroregio -qui a donné son nom générique à toutes celles qui sont nées ultérieurement, surtout après la chute de 
l'empire soviétique-, a marqué le nord rhénan, aux confins belgo-germano-néerlandais. TriRhena a en outre ceci de particulier qu'elle inclut deux portions d'Etats fondateurs de l'Union européenne (Allemagne et France), et une portion d'Etat non communautaire (Suisse).

2 Il est donc utile de rappeler le cheminement qui, en une quarantaine d'années, a suscité la mutation d'un espace marginal en un espace central, formation et développement d'un territoire du savoir à l'appui (Wackermann, 1988 b). Un cheminement auquel la communauté scientifique géographique a participé très activement, dès la "période première " (Wackermann, 1984 e), tant par ses réflexions que par ses propres efforts de construction transfrontalière dans les territoires appropriés du bassin rhénan.

Dans cette démarche, nous analyserons d'abord la situation initiale à laquelle les pionniers de part et d'autre des frontières ont dû faire face, pour présenter ensuite les premières initiatives, avant de mettre l'accent sur l'intérêt de l'importance de l'institutionnalisation, puis de celle de la mise en réseaux.

4 Le présent aperçu est centré sur l'approche faite dans le cadre de la Rencontre d'aménageurs du Rhin Supérieur (« Konferenz oberrheinischer Regionalplaner »), dont j'étais le membre fondateur français, à l'initiative du groupe allemand qui, sous l'impulsion de l'ancien Landrat de Bad Bergzabern, Becker-Marx (Palatinat), a réuni l'universitaire-géographe Werner Fricke, de Heidelberg, et le maire de cette ville, devenu président du nouveau mouvement. Du côté suisse, fut associé le secrétaire général et «l'âme » de la « Regio basiliensis », Hans Briner (Briner, 1974), et, quelque peu plus tard, l'universitaire-géographe bâlois d'origine allemande, Hartmut Leser (Leser, 1977 et 1980/81).

Figure 1 : Périmètre de la Rencontre d'aménageurs du Rhin supérieur

Konferenz Oberrheinischer Regionalplaner Rencontre des Aménageurs du Rhin Supérieur

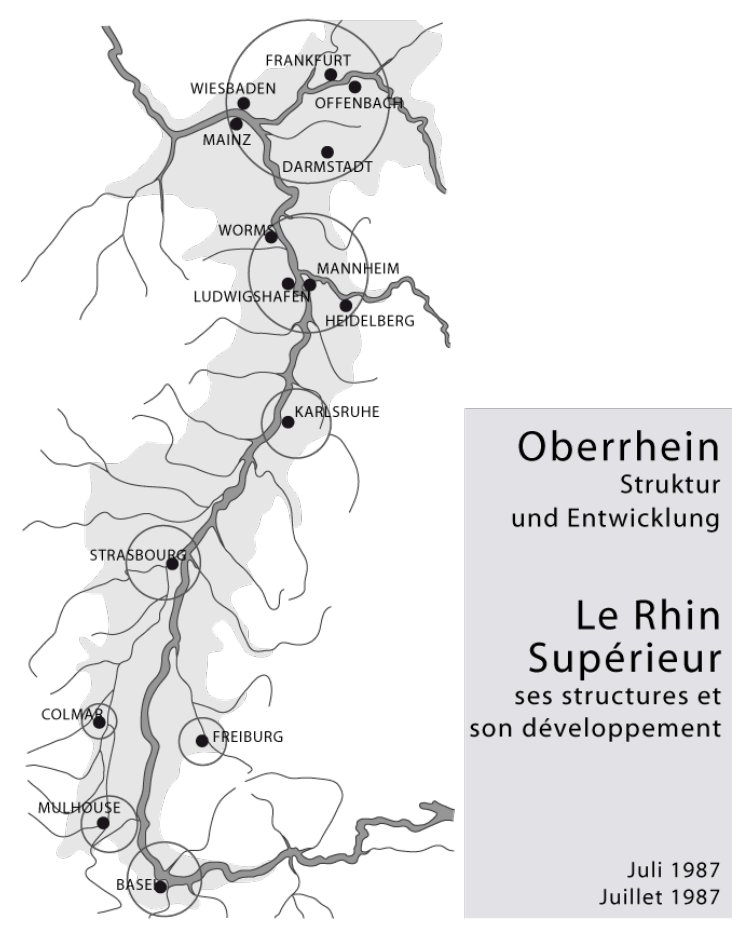


5 En Alsace, l'administration et les collectivités frontalières françaises n'ont adhéré que tardivement à cette initiative, leur premier « porte-parole » officieux, le jeune et très audacieux sous-préfet Pfister -un Alsacien- ayant essuyé les plâtres à la tête de l'OEDAAlsace, à tel point qu'il fut vite déplacé dans la région azuréenne pour s'occuper à Nice d'un organisme international orienté vers la protection et la promotion environnementales. Même Etienne Juillard m'a aimablement conseillé initialement de ne pas choisir l'Allemagne d'en face comme terrain de recherche; il est vrai que des universitaires strasbourgeois influents voyaient encore d'un mauvais œil, durant les années 1950, la main tendue vers l'Allemagne. Mon directeur de thèse d'Etat m'a toutefois laissé faire sans a priori, me recommandant aussitôt auprès du professeur Annaheim (Bâle) et de l'un et l'autre de ses collègues à Heidelberg.

6 En Lorraine par contre, le géographe universitaire de Metz, François Reitel -un ami que Juillard avait mis en garde également-, et en Sarre, le géographe-universitaire Wolfgang Brücher, à Sarrebruck, avec lequel j'ai créé le Groupe universitaire de géographes franco- et germanophones, ont contribué à initier eux aussi les premiers pas actifs interfrontaliers de l'espace sud-rhénan du savoir.

7 Aux sources de documentation relatives à cette ambiance, j'ai intégré ma propre mémoire de pionnier, un apport d'autant plus intéressant et adéquat que dans mon itinéraire alsacien j'ai pu observer la manière dont la France de l'Est s'est intégrée, non sans écueils, à l'Europe rhénane. L'Alsace était, en effet, la région qui avait les plus gros retards socio-économiques à rattraper, une Alsace qui demeure en retrait sur les avantages acquis par l'Allemagne et la Suisse, ayant été pendant longtemps défavorisée par le régime d'excessive centralisation de son Etat. Les références à mes publications, précisées dans ma contribution à l'appui de mes témoignages, sont destinées à resituer dans son ensemble la problématique vécue, subie, puis de plus en plus maîtrisée sur la rive gauche du Rhin d'essence française, au regard de l'évolution rhénane générale.

\section{Similitudes et contrastes initiaux - Atouts et pierres d'achoppement : les prémices}

8 La question de la coopération interfrontalière s'est d'abord posée à l'échelle de la politique générale, conduite par des organismes extérieurs à l'espace du Rhin supérieur encore traumatisé par l'Histoire, surtout du côté français. La mise en place, dès les années 1950, d'associations d'aménagement contribue à sensibiliser l'opinion aux nécessités d'un rapprochement interfrontalier. A cette époque, des contacts directs, notamment avec l'Alsace, demeurent cependant encore sporadiques et d'ordre très personnel (Wackermann, 1969). Ils commencent timidement en milieu politique ami, avec la région bâloise, quoique les dirigeants politiques bas-rhinois, le président Pierre Pflimlin en tête - haut-rhinois de surcroît-, n'apprécient guère ce qu'ils considèrent comme un mouvement régional centrifuge.

9 Ailleurs en Alsace, ce sont des aménageurs et des universitaires allemands du Rhin supérieur qui prennent discrètement les premiers contacts. Le mouvement européen, dirigé surtout par des enseignants, s'est mis également de la partie. La promotion du savoir et du savoir-faire apparaît donc dès l'origine comme une préoccupation interfrontalière pressante, avant qu'elle ne devienne transfrontalière. A l'Institut de géographie de Strasbourg, sous l'impulsion d'Etienne Juillard, l'intérêt est porté de 
façon permanente sur le déploiement urbain (Rimbert, 1965 a et b). Dès 1964, Sylvie Rimbert fait état des «interférences des zones d'influence des grandes métropoles régionales frontalières, françaises et étrangères, des Alpes à l'Ardenne, dans la perspective d'une ouverture européenne des frontières", impliquant par là même notre périmètre (Rimbert, 1964), une recherche pour laquelle j'ai réalisé et fait réaliser de multiples enquêtes. Des préoccupations économiques, ainsi que le souci d'améliorer les conditions sociales se mêlent très vite aux nouvelles ambitions du savoir, les unes étant étroitement imbriqués dans les autres.

L'Alsace est particulièrement handicapée en matière de niveau scolaire et professionnel, apparaissant, dans de multiples secteurs territoriaux, davantage que les régions voisines -allemandes et suisse- comme une pépinière de main d'œuvre peu qualifiée et trop peu scolarisée au-delà du niveau de l'école primaire (Wackermann, 1956, 1958, 1960, 1964). Le nombre de bénéficiaires de l'enseignement secondaire est largement insuffisant et celui de l'enseignement supérieur, notamment technique, trop faible pour faire face aux amples défis d'un monde qui, au lendemain de la Seconde guerre mondiale, a basculé vers la nécessité de compétences affirmées. Le fascicule de références de mes écrits relatifs à cette question permet aux lecteurs que le souhaitent de saisir de près cette situation de marasme culturel, puis l'évolution qui s'est ensuivie à la faveur des prises de conscience et des démarches d'aménagement (Wackermann, 2009).

11 De plus, le modèle de formation national français, trop sélectif et intellectuellement spéculatif, trop peu " applicable » au monde du travail appelé à être de mieux en mieux qualifié, convient très peu aux exigences de compétitivité qualitative rhénane, dont l'Allemagne et la Suisse alémanique sont les heureux bénéficiaires depuis déjà un siècle ou presque (Wackermann, 1978). Même les écoles d'ingénieurs diplômés y bénéficient de passerelles permettant aux apprentis des métiers d'accéder, par le mérite, au niveau de l'ingénieur hautement qualifié. Durant l'Annexion, Strasbourg, alors capitale du «Reichsland » disposait ainsi d'une Ecole technique supérieure de ce genre, supprimée ultérieurement, dans son esprit et sa vocation, par l'administration centralisatrice française, qui a contribué par là même à l'accumulation des retards alsaciens dans le domaine du savoir-faire. Il en est de même de la pratique de l'enseignement professionnel alternatif -mi-scolaire mi-entrepreneurial- propre aux territoires de langue allemande, que l'entêtement centralisateur parisien a supprimé dans les années 1950 en Alsace-Moselle.

12 Est venue, en outre, se greffer sur ces insuffisances, dès la fin des années 1970, la nécessité de formations panachées, pluri- et transdisciplinaires, de même que de recherches du même ordre, en vue de l'adaptation aux nouveaux besoins des entreprises et des administrations, publiques et privées; des profils d'emplois innovants ont impliqué d'urgence des adaptations de l'appareil éducatif à tous les niveaux de la formation générale et professionnelle (Wackermann, 1979 c). Les lenteurs imposées à la création de nouvelles filières, en particulier du côté alsacien pris davantage au dépourvu qu'en pays allemand et suisse, où l'on était habitué de longue date aux adaptations de cette nature, ont contribué à réduire plus encore les capacités concurrentielles du partenaire français.

13 Si, entre 1960 et 1972 les responsables bâlois et mulhousiens accentuent leurs contacts, des personnalités politiques de part et d'autre de la frontière franco-allemande suscitent individuellement des coopérations transfrontalières : Colmar et Fribourg-en- 
Brisgau commencent à être liées par la "Communauté d'intérêts Fribourg-Moyenne Alsace " («Interessengemeinschaft Freiburg-Mittleres Elsass»); dans le nord, entre l'arrondissement de Wissembourg et l'extrême-sud du Palatinat surgit un organisme de réflexion commune, tant économique que culturelle. Des universitaires, de part et d'autre du tracé frontalier, se penchent sur les symptômes du renouveau, et tout spécialement sur le rôle d'entraînement des villes qui polarisent le savoir et le savoirfaire (Reitel, 1966, 1967 ; Traband, 1966, 1969, 1970 ; Lang, 1974).

Les yeux ne commencent vraiment à se dessiller qu'à partir de la fin des années 1960 . Les contacts informels se multiplient. La route reste néanmoins sinueuse. Ce n'est qu'en 1972 que la Rencontre d'aménageurs du Rhin supérieur parvient à réunir à Heidelberg des responsables de la quinzaine d'organismes publics d'aménagement allemands, français et suisses, qui desservent ce périmètre. Mais aucun des présents n'entend encore engager son organisation ni, surtout, les pouvoirs publics. Lors d'un nouveau colloque transfrontalier qui s'est tenu à l'Université de Heidelberg, le 23 novembre 1982, à l'initiative de la même Rencontre, une Recommandation salue la détermination des trois Etats concernés de mettre en œuvre une Commission Tripartite compétente pour le Rhin supérieur. Mesurant les enjeux européens du bassin rhénan méridional dans son ensemble, les auteurs de ce texte formulent en outre le souhait de la création d'une institution publique analogue en ce qui concerne le périmètre frontalier francogermano-luxembourgeois adjacent. Dès le départ, ils ne conçoivent pas une telle entreprise transfrontalière sans la participation active des milieux scientifiques.

Quoi qu'il en soit, dès les années 1970 se dessine la carte des pôles universitaires et de recherche. Les centres de gravité des sciences de l'ingénieur, de la physique et de l'informatique sont localisés à Darmstadt et Karlsruhe, ceux de la biologie et de la médecine à Bâle, Francfort, Heidelberg, Fribourg-en-Brisgau et Strasbourg, ceux de la chimie à Bâle, Francfort, Mayence, Mulhouse et Strasbourg. A Colmar, s'est conforté un centre de recherche d'œnologie et d'agronomie. Les principaux pôles d'innovation en général sont alors -sans souci de classement par ordre d'importance-, du nord au sud, les villes universitaires de Francfort, Mayence, Darmstadt, Heidelberg, Karlsruhe, Strasbourg, Fribourg, Mulhouse et Bâle (Konferenz oberrheinischer Regionalplaner, 1987, p. 19 et 21).

16 Le constat des insuffisances et nuisances de toute nature, aggravant les déséquilibres régionaux et les contrastes entre les trois portions de territoires nationales est en effet éloquent : aux distorsions économiques et sociales, aux contraintes culturelles s'ajoute à partir du début des années 1970 la prise de conscience écologique, activée par l'ambiance très turbulente de 1968, à la fois dans certaines universités rhénanes et dans la rue; une contestation propice à une nouvelle réflexion sur la finalité sociétale. L'opportunité d'un renforcement de la solidarité transfrontalière s'est clairement exprimée, en milieu estudiantin en particulier, universitaire et enseignant en général. A Strasbourg, le Groupe interuniversitaire "Ecologie et éthique » s'est penché dès la seconde moitié des années 1970 sur les rapports du nucléaire à l'espace géographique (Wackermann, 1978 a), sur l'industrialisation et l'exploitation de la nature (Wackermann, 1979 a),... Dans ce domaine, la réorientation souhaitée par les associations concernées appuyées sur des universitaires avisés, porte notamment sur la défense de l'agriculture conservatrice du patrimoine, la protection paysagère, la sauvegarde des milieux fragiles ou rares, la lutte contre les pollutions et nuisances; s'est précisée aussi la critique du système économique. La Rencontre d'aménageurs du 
Rhin supérieur, constatant que le bassin rhénan forme un tout au point de vue de sa protection naturelle, demeure présente et vigilante (Wackermann, 1984 a). L'eau, parce que fort menacée par les excès consuméristes, industriels, agricoles et sociétaux, fait l'objet de préoccupations insistantes. Aussi suscite-t-elle la création de multiples incitations à la recherche, parmi lesquelles il convient d'évoquer l'initiative française à vocation transrhénane, PIREN-Eau/Alsace, à partir de la seconde moitié des années 1970 (PIREN, 1984), en liaison avec la Commission internationale des irrigations et drainages (Wackermann, 1982, 1984 c, 1984 d, 1986 d).

17 Notons -et c'est encore un signe fort de l'extraordinaire imbrication mutuelle des facteurs sur laquelle se fonde l'actuelle société du savoir- que c'est la société de consommation qui a accéléré la prise de conscience de l'intérêt de la protection de l'eau. Elle a même contribué à esquisser une réflexion générale sur les questions hydrologiques, jusqu'à conforter la concertation régionale. A présent, la crise de croissance économique et démographique favorise la régularisation des rapports entre les acteurs sociaux et renforce, dans une certaine mesure, l'influence de l'industrie écologique, seule issue pertinente pour cet espace rhénan surencombré.

La société du savoir a ainsi commencé une mutation décisive, dans la mesure où ce fut l'esprit par lequel ce savoir devait se décliner dorénavant qui a été modifié sensiblement. En même temps, s'est opéré le passage déterminant du savoir traditionnel au savoir postmoderne, faisant basculer notre périmètre vers son évolution plus intense en direction des réseaux de compétence appuyés sur l'appareil universitaire et sur celui de la recherche de facture transfrontalière.

C'est bon gré mal gré que l'on s'achemine, par la force des événements, vers une autre conception de l'organisation sociétale et territoriale. Les technologies y contribuent prioritairement. La course au savoir devient un impératif premier. Les énergies de remplacement ne rendent plus aussi urgentes les constructions de centrales nucléaires programmées. Les eaux chaudes du sous-sol rhénan s'annoncent prometteuses; l'énergie géothermique constitue un espoir pour ce périmètre du bassin du Rhin soumis aux nuisances de l'industrie chimique et des effluents de la potasse.

20 L'écologie, pratiquée à l'université, devenue politique, commence à mettre les pendules à l'heure. Les scientifiques sont devenus indispensables. La raréfaction progressive des ressources financières publiques -le premier choc pétrolier et le début de la crise économique mondiale du début des années 1970 suivent de très près la contestation de 1968-, affaiblissent le processus d'aménagement, au moment même où les bases mathématiques et économiques de celui-ci font l'assaut de critiques croissantes. La crédibilité des aménageurs laisse à désirer; les méthodes d'industrialisation en cours subissent des révisions déchirantes. Nous ne luttons pas seulement contre les nuisances, mais aussi contre les mythes de l'industrialisation ", déclare alors Roland Carbiener, de l'Université Louis-Pasteur de Strasbourg.

21 Le nouveau " discours " géographique se précise, remplaçant celui d'avant 1965 encore fortement teinté de nationalisme, nuançant à la lumière de l'évolution concrète des choses les perceptions de l'espace transfrontalier rhénan. A Bâle, après l'universitaire Annaheim, Werner Gallusser (Gallusser, 1994) et Hartmut Leser (1977, 1980/81) contribuent à animer à cet effet le périodique "Regio Basiliensis ». A Strasbourg, Etienne Juillard élabore des projections métropolitaines qui ne tardent pas à être reprises dans les discussions économiques et politiques, avant de constituer des bases 
de réflexion pour les institutions transfrontalières mises en œuvre (Juillard, 1961, 1968, 1969). Le ton est donné, irréversible.

\section{Débuts de l'institutionnalisation}

La Rencontre d'aménageurs du Rhin supérieur a eu le mérite d'éveiller les consciences ; elle a continué à accompagner les institutions progressivement mises en place (Konferenz oberrheinischer Raumplaner, 1979).

L'OEDA est créée en 1972, une émanation régionale de la DATAR, tout en s'insérant dans la mouvance du ministère de l'Equipement; placée sous la tutelle directe du préfet de région, son comité de direction comprend pour moitié des représentants de l'Etat, pour moitié des élus alsaciens. Elle est aussi chargée d'une mission de coopération avec la Lorraine et la Franche-Comté. Elle doit toutefois se contenter, en matière interfrontalière, d'analyses destinées à déceler les insuffisances alsaciennes, allemandes et suisses dans le périmètre sud-rhénan. Ces analyses sont courageuses, à tel point que le directeur de l'OEDA, le sous-préfet Pfister, déjà évoqué, doit se contenter d'assister comme observateur aux initiatives de la Rencontre d'aménageurs du Rhin supérieur ; très vite, il constate qu'il vaut mieux y déléguer une adjointe qui n'a aucune garantie d'être soutenue en ce qui concerne les projets élaborés par cette Rencontre.

Les freins viennent surtout du côté du gouvernement français. Mais en 1971, se tient la première Conférence tripartite de coordination régionale qui réunit les représentants des trois Etats compétents, ainsi que les élus responsables du sud du périmètre : HautRhin, les deux cantons de Bâle, ainsi que le Bade-Sud. Cette Conférence est remplacée en 1975 par un Comité régional tripartite qui donne naissance à la Commission intergouvernementale chargée de «faciliter l'étude et la solution des problèmes de voisinage dans les régions frontalières » en question. Sont issus de cette Commission le Comité Tripartite franco-germano-suisse, de même qu'un Comité Bipartite francoallemand chargé du Palatinat méridional, du Pays de Bade septentrional, du Pays de Bade méridional et de la région Alsace. Une répartition fonctionnelle des compétences est préférée à une répartition purement géographique. Les enchevêtrements sont complexes, signes d'une forte volonté de tutelle des gouvernements nationaux, surtout de celui de la France (Arnold-Palussière, 1979). Il faut attendre la décentralisation française, intervenue à l'occasion de l'accession de François Mitterrand à la présidence de la République, en 1981, pour assouplir petit à petit la collaboration transfrontalière. En dépit des pesanteurs politiques et des lourdeurs comportementales en milieu sociétal et entrepreneurial, particulièrement en Alsace, les tenants du savoir occupent petit à petit une place suffisante pour accélérer la dynamique institutionnelle (Becker-Marx \& Fricke, 1981; Kistenmacher, 1982).

Figure 2 : L'organisation des régions transfrontalières en Europe centrale 


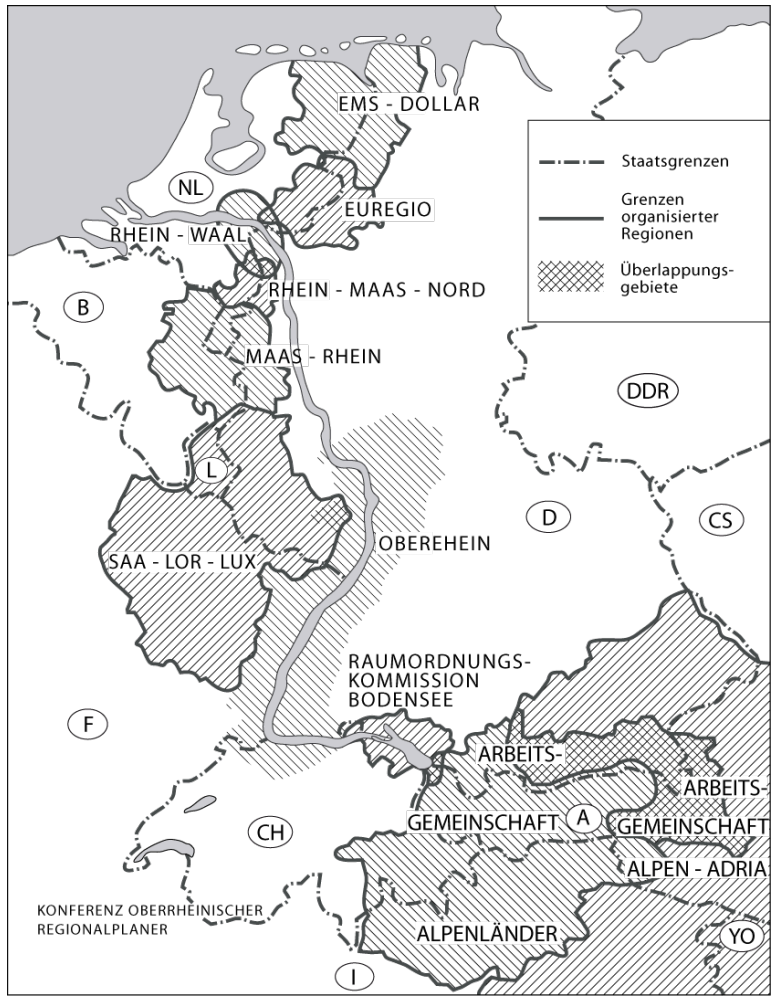

La figure 2 montre que toutes les régions transfrontalières sont institutionnalisées et nettement délimitées, à l'exception du périmètre du Rhin supérieur qui, en outre, comporte dans sa partie septentrionale une aire transrhénane non frontalière, mais qui est fortement ancrée tant dans la problématique urbaine que dans la polarisation territoriale induite par les réseaux de villes (Wolf, Schymik, Jurczek, 1983), le tout à base de forte innovation technologique, d'un tertiaire hautement qualifié en savoir et savoir-faire (Wackermann, 1988 a). C'est ce que Christaller avait déjà entrevu et cartographié dans son ouvrage fondamental intitulé "Die zentralen Orte in Süddeutschland» (édité à Iéna en 1933).

Il s'agit là d'un périmètre fort original et souple, très diversifié dans ses composantes, doté d'atouts qui, depuis des décennies, n'attendent qu'à être valorisés, une opportunité offerte par l'entrée de celui-ci dans l'ère technologique. C'est le cas tant de ses milieux urbains que de ses campagnes. Dès la fin du XIX e siècle, les nombreux réseaux urbains, la densité des villes, les grands courants de communication stimulés par de puissants carrefours de toute nature, ont accéléré les tendances au changement. La vitalité du monde agricole rhénan, les relations étroites entre villes et territoires ruraux, le désir d'une minorité de paysans -les riches- de participer au mieux aux innovations urbaines ont rendu les milieux villageois aisés très sensibles aux progrès techniques générateurs de rendement, de richesse et de bien-être. Ici, le progrès était surtout induit par les nouvelles machines qui ont facilité le travail de la terre, le conditionnement et la transformation des produits, ainsi que le recours aux engrais chimiques. S'inspirant de procédés américains, inventant à leur tour des méthodes de fabrication, des firmes spécialisées européennes, dont bon nombre étaient localisées dans l'espace rhénan dès la fin du XIX e siècle ou le début du XX e siècle, ont cherché à susciter des applications multiples dans des régions particulièrement orientées vers l'acquisition de moyens 
techniques destinés à renforcer leur puissance économique et leur emprise socioculturelle.

Fortes de cette résonance en milieu agricole aisé, les entreprises de matériels et matériaux agricoles ont utilisé les canaux publicitaires appropriés à chaque période historique : foires-expositions et démonstrations locales dès la fin du XIX e siècle, cours du soir dits " postscolaires ", séances d'initiation pratique en salle scolaire, publique ou confessionnelle, et sur le terrain, action publique et confessionnelle agricole, un ensemble d'activités généralisées à partir du début du XX e siècle. Aux Pays-Bas, en Allemagne et en Suisse, les ministères de l'agriculture respectifs, appuyés par les partis conservateurs ruraux, sont également intervenus pour mieux insérer leurs territoires dans la compétition mondiale. Les régions voisines, alsacienne et mosellane, se sont inspirées assez rapidement des modèles élaborés, d'autant plus que le temps de l'Annexion (1871-1918) était un moment crucial dans l'évolution des techniques et des mentalités. Ainsi, l'Alsace-Moselle (l' « Alsace-Lorraine » officielle), annexée au nouvel empire allemand, devenue «Reichsland» directement rattaché à l'administration prussienne-brandebourgeoise, soucieuse de progrès technique depuis quasiment les débuts de l'époque moderne, a pris des habitudes qu'elle a conservées lors de son retour à la France, mais que sa situation devenue périphérique et de glacis n'a pas autorisé à exploiter suffisamment à partir de 1918. Durant l'Entre-deux-guerres, la généralisation des écoles agricoles, l'influence renforcée de la presse écrite, l'apparition de la presse parlée, la multiplication des affiches murales, le cinéma et ses bandes publicitaires ont véhiculé avec efficacité idées et images fondées sur le progrès technique en agriculture.

Après le second conflit mondial, les moyens de communication sociale s'amplifiant, la télévision intervenant massivement dès 1955, l'information s'est transmise d'autant mieux que les conditions du marché se sont annoncées dures et avérées impitoyables pour les exploitations agricoles fragiles. Une faible partie de celles-ci a toutefois constitué un marché complémentaire pour les firmes industrielles et commerciales : ne disposant ni d'une superficie adéquate ni des capitaux requis, elles ont limité leur activité agricole à l'élevage de petit bétail ou d'animaux de basse-cour; l'acquisition d'installations à technologie élaborée a favorisé le maintien du genre de vie mixte du chef de famille, qui était ouvrier, artisan, petit employé ou commerçant durant les séquences de travail "ouvrable"; le double revenu favorisait les investissements techniques agricoles» et l'ouverture d'esprit nécessaire à la prise de conscience du fait qu'il convenait désormais d'envoyer autant que possible les enfants à l'école secondaire venue se généraliser ou se renforcer dans les campagnes et s'installer dans les gros bourgs et petits centres urbains.

Ce long parcours rural d'un siècle environ, perceptible un peu partout au début de la seconde moitié du XX e siècle à travers de nombreuses structures matérielles et mentales, étroitement connecté au développement urbain, a favorisé les mutations professionnelles et la valorisation du travail de la femme, elles-mêmes fortement liées au mouvement de modernisation général. Partout, dès lors que le tertiaire de l'agriculture a pris des proportions importantes au niveau de la gestion et des activités de services, le monde agricole devenu surtout rural a évolué dans une aisance telle qu'il a disposé de signes extérieurs de richesse ou d'aisance aussi importants que ceux propres aux catégories bourgeoises voisines. Les cas de plus en plus fréquents d'agriculteurs cossus épousant des enseignantes, des assistantes sociales, des infirmières, des employées de bureau, des 
techniciennes et techniciennes supérieures témoignent du parachèvement de la mutation technologique et, par là même, socio-professionnelle (Wackermann, 1981).

L'engrangement de ces avancées rurales, ajoutées à celles des villes, l'attraction rhénane d'un espace devenu central dans une Europe davantage internationalisée, ont en somme modelé le Rhin supérieur méridional de manière qu'il soit déjà, entre autres, la préfiguration de la "Biovalley» transfrontalière de la fin $d u$ XX e siècle. Même si la phase d'institutionnalisation, freinée par les lourdeurs imposées par les pouvoirs publics nationaux, surtout en Alsace, n'a grosso modo pas vraiment contribué à la vraie dynamique socio-économique, elle a permis d'officialiser l'évolution en cours et de conforter le cadre territorial (Speiser, 1993) avec l'appui des gouvernements centraux, surtout face à la Commission de Bruxelles.

\section{Vers la mise en place de réseaux}

31 La mise en réseaux a été accélérée, nous l'avons vu, par l'urbanisation accentuée à la faveur de l'irruption technologique (Wackermann, 1986 e). Déjà, au début des années 1960, le sociologue français, Pierre Naville, conscient des nouveaux enjeux de la région frontalière de l'Est français, face au «miracle économique " allemand et à la montée internationale croissante de la Suisse, m’a demandé de réaliser une étude sur les effets sociaux de l'automation en Alsace (Wackermann, 1961). L'irruption des Nouvelles technologies de l'information et de la communication (NTIC) a renforcé sensiblement l'orientation de notre périmètre vers des exigences d'une tertiairisation de niveau élevé (Wackermann, 1979 b), impliquant une internationalisation croissante des contacts, bousculant ainsi les dernières pesanteurs fondamentales des passivités frontalières (Wackermann, 1984 b, 1986 b, 1987 c, 1988, 1990).

Le périmètre observé dispose d'une densité très confortable de centres d'impulsion qui forment de véritables "nœuds magnétiques", selon l'expression en vogue durant les années 1980. Francfort et Zurich constituent aux deux extrémités de notre aire de vigoureuses places mondiales du savoir et du savoir-faire, voire du savoir-être, prolongées l'une par Mayence, Wiesbaden et Darmstadt, l'autre par le Liechtenstein et le secteur frontalier austro-germano-suisse. Connectées étroitement avec les autres métropoles de leur pays respectif et du Globe en général, elles bénéficient d'un environnement tertiaire favorable: Heidelberg, Stuttgart-Karlsruhe, l'aire métropolitaine bâloise et le triangle "Saar-Lor-Lux » (Brucher, Quasten \& Reitel, 1982) sont des supports qu'à elles seules ni l'Alsace en général ni la Franche-Comté n'ont à mettre en avant. Lyon et Paris continuent ici à jouer un rôle métropolitain lointain, cependant de plus en plus rapproché dans le temps par la télématique et la primauté de la vitesse acquise sur le temps dans le domaine des transports. Sont particulièrement avantagés dans ce réseau de connexions majeures les pôles métropolitains de Hesse, du Bade-Wurtemberg et de la Suisse du nord, habitués de surcroît depuis des siècles à un fonctionnement décentralisé des institutions et des firmes.

La première rencontre des universités du Rhin supérieur, qui a lieu en 1986 au Conseil de l'Europe, porte, dans ces conditions, tout naturellement sur la communication et les transferts de technologie. Suisse et République fédérale d'Allemagne y témoignent d'un savoir-faire indéniable, alors que l'Alsace, notamment Strasbourg, dispose d'un extraordinaire savoir encore trop peu mis en valeur dans une optique transfrontalière. Les retards régionaux sont généralement démultipliés dès lors qu'au niveau qui nous concerne ici 
ressortent les exigences de qualité des télécommunications. L'avance prise par le BadeWurtemberg permet à cette époque au ministre-président, Lothar Späth, d'annoncer que le système de centre de calcul Gray va être mis en place depuis la capitale du Land et desservir l'ensemble des besoins de recherche du Bade-Wurtemberg. Notre homme politique a ajouté que cet équipement est susceptible d'utilisation à des fins transfrontalières. Il a néanmoins signalé que le centre de calcul Gray était pour le moment davantage destiné au niveau d'amples relations avec la métropole lyonnaise qu'avec les régions frontalières environnantes. Par boutade, lorsqu'un interlocuteur lui a demandé en quoi ce centre pouvait renforcer les liens de jumelage de Stuttgart avec Strasbourg, il a répondu qu' "à Strasbourg, on mange bien ». Signalons pourtant, que Stuttgart, capitale d'un Land plus important que la Hesse, demeure coiffée par Francfort, métropole au savoir et savoir-faire plus ample, aux hautes fonctions financières et aux relations supérieures internationales de commandement en matière économique (Wackermann, 1987 c).

Le mouvement technopolitain général qui se met en place (Wackermann, 1987) ne fait que renforcer cette évolution. La télématique induite par les pôles urbains irriguant progressivement les milieux ruraux (Wackermann, 1989; Wackermann \& Paal, 1994) constitue une force d'entraînement de l'ensemble des territoires du périmètre observé. La mobilité croissante des firmes et des gens par suite de la force d'attraction des villes (Wackermann, 1989 a et b) anime avec force et de manière permanente les régions du Rhin supérieur. L'entrée dans la société de consommation à la faveur de l'élévation des niveaux de vie, dans une ambiance rhénane riche en peuplement et en sites de proximité, fait le reste (Wackermann, 1983, 1986 a).

Dans ces conditions de désenclavement, un réel vécu transfrontalier se précise (Wackermann, 1987 a), accentuant le renouveau culturel qui change d'échelle et d'intensité spatiale (Wackermann, 1986 c). Se forge petit à petit une certaine identité " rhénane ", quoique surtout perçue encore en dernier lieu par les habitants, les préférences régionales locales et nationales l'emportant encore de façon courante (Fichtner, 2003). Une réelle ambiance de bien-être matériel et mental s'installe, sous-tendue par la transfrontiérité (Traband, 2008 ; Nonn, 2008). Il convient cependant de rappeler que les sociétés multiculturelles économiquement avancées révèlent que leurs principaux problèmes ne sont plus posés aux frontières politiques, mais au coeur de l'Etat, dans les villes même qui donnent l'impulsion nationale décisive à la vie courante.

Par ailleurs, la combativité socio-économique des voisins allemands et suisses de l'Alsace s'est appuyée pendant longtemps sur les performances technologiques des entreprises de production, un sens remarqué de l'organisation des circuits du marché et l'intégration des seconde ou troisième générations de travailleurs immigré aux principes de commandement du niveau des cadres moyens, garants de la célèbre "Leistung». Si ce profil ne fait que s'accentuer, il ne caractérise toutefois plus principalement les positions de pointe du périmètre considéré. Le " plus » actuel, certes inexplicable en-dehors de l'évolution précédente, est fondé sur les atouts inhérents aux fonctions supérieures d'entraînement fondamental de notre civilisation: la communication et les professions à haute valeur intellectuelle ajoutée, susceptibles, par leur synergie, d'assurer une innovation permanente dans l'ambiance de mobilité accrue de notre époque.

Sur cette lancée, le groupe de scientifiques alsaciens qui m'ont aidé ponctuellement en 1986 à lancer, dans le cadre de la Rencontre d'aménageurs du Rhin supérieur, une 
analyse relative aux possibilités de collaboration interuniversitaire transfrontalière, a permis de constater que l'Université de Karlsruhe avait mis au point plus de 600 projets prêts au transfert de technologies, grâce à deux banques de données (Siemens BS 2000 et MS DOS), exploitables selon environ 1400 mots-clés. Le système BTX de la Deutsche Bundespost avait déjà engrangé une soixantaine des projets précités se rapportant à la Faculté d'électronique. L'Université Louis-Pasteur de Strasbourg disposait d'une documentation semblable à celle de son homologue de Karlsruhe. Elle était en train d'élargir les données à l'ensemble des universités et laboratoires d'Alsace. L'initiative prise était positive, d'autant plus que jusqu'alors il n'existait pas de contacts entre les deux universités en vue de la promotion du transfert de technologie et de relations industrielles réciproques (Wackermann, 1990, p. 78/79).

Pour Strasbourg, le rôle du Parlement européen reste déterminant, d'autant plus que la stratégie de Lisbonne n'a toujours pas réussi à promouvoir suffisamment dans notre périmètre sud-rhénan la dynamisation du triangle de la connaissance (enseignement supérieur, recherche et innovation), les effets de l'Agenda social européen et de l'intégration des marchés financiers demeurant déficients. C'est que l'Alsace, partie territoriale toujours encore fragile dans cette structure transfrontalière, est un handicap dans l'effort consenti par ses partenaires en faveur de l'évolution vers l'optimisation de la mise en réseaux de portée internationale.

Dans le cadre des «Matinées » que j'organise chaque année à Strasbourg, de concert avec des personnalités universitaires et politiques, des responsables régionaux du monde économique, social et culturel, nous avons pu procéder le 21 janvier 2006 à une ébauche de diagnostic fort expressive (Elan, 2006). C'est ainsi que l'économiste J.-A. Héraud s'est interrogé sur le devenir de la région en se demandant ce qu'allait devenir, entre autres, à l'échelle de la gouvernance multi-niveaux dans le domaine de la politique de la science, de l'innovation, des transferts de technologie, de l'éducation et de l'enseignement supérieur, le contrat de plan Etat-Région, « un des rares instruments de coordination multi-niveaux qui fonctionne jusqu'à présent. La décentralisation se construit sur ce type d'outil. S'il disparaît, sur quoi édifiera-t-on cette fonction indispensable de comparer, négocier les 'priorisations' de chacun des acteurs intervenant sur un territoire donné ?».

Dans la foulée, P. Siffert, directeur de recherches honoraires au CNRS, n'a pas hésité à affirmer que «l'avantage des physiciens, c'est qu'ils avancent non par des compromis mais par des conflits d'idées. J'oserai donc dire, avance-t-il, qu'il y a, me semble-t-il, en Alsace, une certaine autosatisfaction, que notre province ressemble un peu à la grenouille qui se prend pour un bœuf. En consultant, en effet, un certain site Internet, je trouve 5 prix Nobel, 120000 employés pour l'industrie automobile; je ne sais où ils sont. Autre perplexité à propos de la création d'un certain nombre de groupes techniques susceptibles de développer l'innovation: les conclusions me semblent orthogonales par rapport à ce qui se passe partout ailleurs dans le monde ».

Le secrétaire général de la CFDT Alsace, B. Marx, quant à lui, a livré sa réflexion avisée sur la présentation du nouveau Schéma régional de développement économique par le président du Conseil régional, qui a mis l'accent, selon lui, «sur une mondialisation venue percuter l'Alsace " plutôt que sur les faits observés. "Nous sommes dans un schéma défensif plutôt qu'offensif », s'est-il empressé d'ajouter. Et de poursuivre : « ... l'un des défauts du même Schéma est que tout part de l'industrie. Aucune référence aux services High tech de la Recherche- développement, ni aux services à la personne. 
L'image de l'Alsace reste celle d'une forte industrie manufacturière au niveau européen en train de disparaittre. Sommes-nous en capacité d'avoir des ambitions, d'afficher des priorités, des choix ? Selon un Conseiller général du Haut-Rhin, depuis juin 2005, 10 millions d'euros ont été votés pour le volet économique. On ne sait toujours pas qu'en faire. On redistribuera donc à qui le demandera. Il n'y a pas de stratégie sur ce plan ».

L'économiste P. Dévoluy a avancé un argument qui vaut son pesant d'or: «En 2003, rapportait-il, j'étais allé voir un responsable local qui m'a demandé de ne pas raconter ce que je lui avais dit. Un article récent du monde m'y incite : je partais de l'hypothèse que le Parlement européen, inéluctablement, allait quitter Strasbourg pour des questions de rationalité économique. Me disant que ce Parlement représentait 200 millions d'euros par an, je proposais l'idée que dans la stratégie de Lisbonne nos très bonnes universités pourraient avec ces 200 millions, permettre à Strasbourg d'avoir une université internationale, en compensation d'une perte importante. Dans les 25 ans, cette université pourrait devenir l'une des cinq premières mondiales ».

Regardons la réalité en face : le seul modèle du savoir étroitement associé au savoirfaire à l'échelon de l'enseignement supérieur professionnel qui a permis à l'Alsace d'émerger a été le «modèle mulhousien » cultivé au XIX e siècle, à l'époque des grands patrons industriels dont on n'a plus guère perpétré le haut niveau de compétence après 1918 et surtout plus après 1945. Dans le nord alsacien, Strasbourg n'est pas une métropole; c'est une grande ville régionale avec des fonctions internationales, notamment européennes. Celles-ci sont toutefois encore insuffisantes pour faire bénéficier la ville d'une forte affirmation (Elan, 2006). L'avenir positif de cette région réside dans le façonnement progressif d'une grande aire métropolitaine transfrontalière sud-rhénane, à même de promouvoir de vraies interconnexions ainsi que des réseaux complets et complémentaires dans les secteurs les plus divers -économiques, universitaires, socioculturels- aptes à l'innovation permanente en rapport avec le restant du globe.

\section{Conclusion}

Le renforcement sensible de la compétence, l'émergence d'une région transfrontalière à savoir et savoir-faire de haut niveau, sous-tendu par l'ambiance d'émulation de l'espace européen directement environnant, ainsi que la constitution de réseaux internationaux d'ampleur mondiale, ont contribué à la multiplication de profils de compétence élevés, sans toutefois exclure le chômage et la pression sur la limitation des rémunérations. Une position qui contraste fortement avec la situation de naguère et qui témoigne de l'immense chemin parcouru, tranchant- en France de l'Est surtoutavec les pépinières de manœuvres d'antan aux qualifications et salaires faibles, au sousemploi latent et au chômage rampant. La reprise progressive de la crise mondiale depuis le début des années 1970 est toutefois venue, aussi sur ce territoire sud-rhénan, interroger de plus en plus la socio-économie du savoir et du savoir-faire qui en a fait un espace de prospérité relative. Désormais incontournable, cette société de promotion de l'intelligence théorique et pratique, bâtie sur la science fondamentale et la science applicable ou appliquée, est appelée à son tour à se remettre en question en fonction des nouveaux enjeux mondiaux qui rejaillissent nécessairement sur les divers territoires du globe. 


\section{BIBLIOGRAPHIE}

Arnold-Palussiere M. (1979). - La coopération transfrontalière régionale en matière d'aménagement du territoire - Etude du cas de la vallée du Rhin, Thèse, Faculté de droit de l'Université de Strasbourg.

Becker-Marx K., Fricke W. (1981). - « Stand der grenzüberschreitenden Raumordnung am Oberrhein ", Heidelberger geographische Arbeiten, cahier 71, Heidelberg Geographisches Institut, $106 \mathrm{p}$.

Briner H.J. (1974). - 5 Beispiele von typischen Schwierigkeiten bei landesgrenzüberschreitender Zusammenarbeit, Bâle, Regio Basiliensis, manuscrit.

Brücher W., Quasten H., Reitel F. (dir.) (1982). - Atlas Saar-Lor-Lux, Saarbrücken-Metz-LuxemburgTrier, Publications de la Commission régionale Sarre-Lorraine-Luxembourg-Rhénanie/Palatinat, 87 p. 4 cartes coul. h.-t.

Elan (Revue) (1986). - Innovations, révolutions technologiques, mais limites des systèmes, Strasbourg, Cahiers du FEC, p. 9-16.

Fichtner U. (2003). - « Architekturen grenzüberschreitender Kooperation und raumbezogene Identität am Oberrhein », Europa Regional, p. 102-117.

Gallusser W. (dir.) (1994). - Political boundaries and coexistence - Proceeding of the IGU-Symposium Basle, Bâle.

Juillard E. (1961). - Dimensions spatiales et démographiques des régions de développement de l'Europe, Lausanne, Université.

Juillard E. (1968). - L’Europe rhénane - Géographie d'un grand espace, Paris, A. Colin.

Juillard E. (1969) - « L'Alsace va-t-elle basculer dans l'orbite économique allemande?», Strasbourg, Commission de liaison Université-Economie, Chambre patronale des industries du Bas-Rhin, manuscrit ronéoté du 21 février.

Kistenmacher H. (1982). - Kurzbericht zum Forschungsprojekt: Möglichkeiten und Restriktionen der grenzübergreifenden Abstimmung der Siedlungsstruktur- und Raumwirkungskonzeptionen am Beispiel des Raumes Südpfalz/Elsass/Mittlerer Oberrhein, Université de Kaiserslautern, manuscrit.

Konferenz oberrheinischer Regionalplaner (Rencontre d'aménageurs du Rhin supérieur) (1987). Le Rhin supérieur ses structures et son développement, Karlsruhe, $80 \mathrm{p}$.

Konferenz oberrheinischer Regionalplaner (Rencontre d'aménageurs du Rhin supérieur) (1979). L'aménagement par delà les frontières - Le couloir rhénan, Bonn, Europa Union Verlag, 86 p.,11 cartes en couleur h.t.

Lang S. (1974). - Der Beitrag der Regionalplanung und der Raumordnungspolitik zu raumbedeutsamen Entscheidungen in Grenzräumen, dargestellt am Beispiel der Agglomeration von Strasbourg und dem Mittelbadischen Grenzraum, Thèse, Université de Stuttgart.

Leser H. (1977). - Bedeutung der natürlichen Ressourcen im Oberrheingebiet, Heidelberg, Konferenz oberrheinischer Regionalplaner, Manuskript.

Leser H. (1980/81). - Raumordnung Oberrheingraben, Cours, Geographisches Institut, Université de Bâle.

Nonn H. (2008). - L'Alsace et ses territoires, 2008, Strasbourg, Presses universitaires, 576 p. 
PIREN-Eau/Alsace (1984). - Synthèse des recherches, Strasbourg, CNRS.

Reitel F. (1966). - Les régions de la France de l'Est et leur environnement géographique, Strasbourg, Crédit mutuel de l'Est.

Reitel F. (1967). - « Karlsruhe - Une fantaisie princière du XVIIIe siècle devenue métropole rhénane », Bulletin de la Faculté des Lettres, Strasbourg.

Rimbert S. (1964). - Les interférences des zones d'influence des grandes métropoles régionales frontalières, françaises et étrangères, des Alpes à l'Ardenne, dans la perspective d'une ouverture européenne des frontières, Rapport à la Commission nationale d'aménagement du territoire (11 avril), Strasbourg.

Rimbert S. (1965a). - « Frontières et influences urbaines dans la 'Dreiländerecke' », Regio Basiliensis, VI,1, p. 35-57

Rimbert S. (1965b). - « Strasbourg, métropole d'équilibre ?», Regio basiliensis.

Schneider-Sliwa R. (dir.) (2003). - Regio TriRhena und südlicher Oberrhein: Ein Raum ohne Grenzen?, Bâle, Institut für Geographie, coll. „Basler Stadt- und Regionalforschung“, vol 22.

Speiser B. (1993). - Europa am Oberrhein: der grenzüberschreitende Regionalismus am Beispiel der oberrheinischen Kooperation, Bâle, coll. „Schriften der Regio“, vol. 13.

Traband A. (1966). - Villes du Rhin, Strasbourg et Mannheim-Ludwigshafen, Strasbourg, Publications de la Faculté des Lettres.

Traband A. (1969). - « De l'influence du Rhin-Frontière sur l'organisation régionale », Bulletin de la Faculté des Lettres, Strasbourg.

Traband A. (1970). - « Grenzprobleme und Raumordnung am Oberrhein », Wiesbaden, Geographische Zeitschrift, 2, p. 124-137.

Traband G. (2008). - Effacer la frontière ? Soixante ans de coopération franco-allemande en Alsace du Nord, Strasbourg, Ed. La Nuée bleue, 287 p.

Wackermann G. (1956). - « Profession et formation professionnelle dans le Nord de l'Alsace », Publications du Comité pour l'Economie bas-rhinoise, Strasbourg.

Wackermann G. (en collaboration avec E. Juillard) (1958). - « Les perspectives de l'industrialisation dans le Bas-Rhin », Bulletin de la Faculté des Lettres de Strasbourg.

Wackermann G. (1960). - « Les problèmes de l'emploi industriel à Wissembourg (Bas-Rhin) », Publications du Comité pour l'Economie alsacienne, Strasbourg, $10 \mathrm{p}$.

Wackermann G. (1961). - « Les effets sociaux de l'automation en Alsace », Les conséquences sociales de l'automation, Paris, Conseil supérieur de la recherche scientifique.

Wackermann G. (1964). - Jeunesse professionnelle et jeunesse scolaire dans le Bas-Rhin, Strasbourg, Institut de promotion et de recherches sociales et familiales d'Alsace, $17 \mathrm{p}$.

Wackermann G. (1969). - « Die Region Südpfalz - Chancen eine grenzüberschreitenden Zusammenarbeit », Universitätsseminar Kandel, Karlsruhe, 15 p.

Wackermann G. (1978a). - Le nucléaire et l'espace géographique, Strasbourg, Groupe interuniversitaire « Ecologie et éthique », CERIT, Faculté des Lettres et Sciences humaines, Actes du Séminaire du 25.4.1978.

Wackermann G. (1978b). - « Profil de l'emploi et de la main-d'œuvre dans le tertiaire décentralisé de l'Est français », Analyse de l'espace, no. Spécial « Décentralisation des activités tertiaires », cahier 4, p. 31-37. 
Wackermann G. (1979a). - L'industrialisation et l'exploitation de la nature, Strasbourg, Groupe interuniversitaire « Ecologie et éthique », CERIT, Faculté des Lettres et Sciences humaines, Téléenseignement 1976/77, Série « Les sciences, la société et la foi devant le défi écologique ».

Wackermann G. (1979b). - « Frontière et échanges tertiaires dans l'espace rhénan au XX e siècle », Actes de 103e Congrès national des Sociétés savantes, 10-15/4/1978 (Nancy-Metz).

Wackermann G. (1979c). - « Le problème des formations panachées et la recherche, au niveau des entreprises, de la définition de nouveaux profils », Actes du Stage de formation des Inspecteurs de l'Information et de l'Orientation de l'Académie de Nancy-Metz.

Wackermann G. (1981). - « La diffusion des progrès en milieu agricole dans l'espace rhénan au XXe siècle ", Actes du 105e Congrès national des Sociétés savantes, CNRS, Comité des travaux historiques, section Géographie, Paris, Ed. Bibliothèque nationale, p. 311-322.

Wackermann G. (1982). - « Géographie historique des relations entre la société et l'eau en Alsace », Saisons d'Alsace, cahier 77, Strasbourg.

Wackermann G. (1983). - « Mutations récentes dans le Nord et le Midi alsaciens, Outre-Forêt et Sundgau », Histoire de l'Alsace rurale, Strasbourg, Ed. Istra, p. 473-488.

Wackermann G. (1984a). - « Nuisances et propositions d'aménagement en espace industrialisé Le cas de la Conférence d'Aménageurs du Rhin supérieur ", Bulletin de l'Association de géographes français.

Wackermann G. (1984b). - « L'impératif technologique pour l’Alsace », Elan, n³-4.

Wackermann G. (1984c). - « Le rôle de l'eau dans les implantations industrielles en Europe rhénane et centrale depuis 1945 ", Revue géographique de l'Est, n²-3, p. 207-213.

Wackermann G. (1984d). - « Les différenciations spatiales du comportement face à l'eau en Alsace et à la formation du consensus ou de la dialectique sociale ", Synthèse des recherches, Strasbourg, PIREN-Eau/Alsace.

Wackermann G. (1984e). - « Chronique rhénane I », Revue géographique de l’Est, n4, p. 339-349.

Wackermann G. (1986a). - « Régimes alimentaires et force de travail - L'exemple de l'espace transfrontalier du Rhin supérieur depuis l'ère industrielle », Revue géographique de l'Est, édité en 1989 aux Presses universitaires de Nancy dans Alimentation et régions.

Wackermann G. (1986b). - L'impératif technologique et la collaboration transfrontalière rhénane - Ses structures et son développement, Karlsruhe, Ed. Rencontre d'Aménageurs du Rhin Supérieur, pp. $17-26$

Wackermann G. (1986c). - « Nouvelles dimensions de l'observation culturelle en espace transfrontalier », Bulletin de l'Association de Géographes Français, n², pp. 131-140

Wackermann G. (1986d). - « Eau et comportement social dans l'espace du Rhin Supérieur », Premier colloque scientifique des universités du Rhin Supérieur : recherches sur l'environnement dans la région (Strasbourg, 27-28.6.86), Proceedings édités par la Conférence des Recteurs et Présidents des Universités du Rhin Supérieur, pp. 940-946

Wackermann G. (1986e). - Belfort, Colmar, Mulhouse, Bâle, Fribourg-en-Brisgau - Un espace économique transfrontalier, Paris, La Documentation française, coll. « Notes et études documentaires », no. 4824, $144 \mathrm{p}$.

Wackermann G. (1987a). - « Le vécu transfrontalier et ses répercussions », Revue géographique de l'Est, n¹-2, pp. 5-18. 
Wackermann G. (1987b). - « Technopoles et compétition internationale en Alsace », Nouveaux dynamismes régionaux - Cahiers du GS 20 - CNRS, $n^{\circ} 1$, pp. 139-150.

Wackermann G. (1987c). - « La combativité transfrontalière et l'importance des technologies de communication dans la dynamique urbaine : l'exemple du Rhin supérieur ", Comité des travaux historiques, Section de géographie, CNRS, 112e Congrès national (Milieux, villes et régions), p. 385-397.

Wackermann G. (1988a). - « L'espace frontalier alsacien face aux nouvelles technologies », Cahiers du GS 20-CNRS, n², pp. 83-88

Wackermann G. (1988b). - Problématique de l'aménagement du territoire dans l'espace multinational du Rhin supérieur jusqu'en 1983, Mulhouse, Publications du Laboratoire de recherches internationales en transport et échanges (LARITE), Université de Haute-Alsace, 436 p.

Wackermann G. (1989a). - « La mobilité des firmes et ses effets sur la population active L'exemple de l'espace transfrontalier du Rhin supérieur », Bulletin de l'Association de Géographes Français, $\mathrm{n}^{\circ} 2$, pp. 103-109

Wackermann G. (1989b). - « Transports et comportements - Une problématique en espace multinational : le cas du Rhin supérieur méridional », Revue géographique de l’Est, n³-4, pp. 187-204

Wackermann G. (1989c). - Der ländliche Raum im Elsass vor den Veränderungen durch den Europäischen Binnenmarkt, Stuttgart, Dokumentation, Arbeitskreis IV - Fachtagung Ländlicher Raum, 6/7.9.1989 in Bad Rappenau, Ministerium für Ländlichen Raum, Baden-Württemberg, pp. 81-93.

Wackermann G. (1990). - Les interactions de la communication et des activités tertiaires dans l'espace du Rhin Supérieur, Paris, IDATE, Communications et territoires, Ed. La Documentation Française, pp. 71-81.

Wackermann G, Paal M. (1994). - Telematik und ländlicher Raum, Paris, Institut de géographie, Université de Paris-Sorbonne, 204 p. + annexes.

Wackermann G. (2009). - Références générales de Gabriel Wackermann (Edition augmentée mise à jour en 2009), Strasbourg, 57 p. ; exemplaire déposé à la Bibliothèque nationale et universitaire de Strasbourg : voir rubrique « Articles de G. Wackermann », p. 26-48.

Wolf K., Schymik F., Jurczek P. (dir.) (1983). - Der Verdichtungsraum in Regionalforschung und zukünftiger Raumordnung - Beispiel Rhein-Main-Gebiet, Francfort, Rhein-Mainische Forschungen, cahier 98, $191 \mathrm{p}$.

\section{RÉSUMÉS}

Il est utile de rappeler le cheminement qui, en une quarantaine d'années, a suscité la mutation d'un espace marginal en un espace central, formation et développement d'un territoire du savoir à l'appui. Un cheminement auquel la communauté scientifique géographique a participé très activement, dès le début, tant par ses réflexions que par ses propres efforts de construction transfrontalière dans les territoires appropriés du bassin rhénan.

Le renforcement sensible de la compétence, l'émergence d'une région transfrontalière à savoir et savoir-faire de haut niveau, sous-tendu par l'ambiance d'émulation de l'espace européen directement environnant, ainsi que la constitution de réseaux internationaux d'ampleur mondiale, ont contribué à la multiplication de profils de compétence élevés. 
It is useful to review the progress which, in forty years, has transformed a marginal space in a central space through the formation and development of a knowledge base. A progress in which, since the beginning, the geographical community was a very active participant mostly through its ideas and own efforts towards cross-border construction in appropriate territories of the Rhine basin.

The strengthening of competence, and the emergence of a cross-border region with a high knowledge base is reinforced by the atmosphere of the surrounding European space just as international networks have contributed to the increase in high levels of competence profiles.

In diesem Heft ist es sicher nützlich an den vierzigjährigen langen und schwierigen, strapazenreichen Weg zu erinnern, welcher von einem marginalen Grenzraum zu einer zentralen grenzüberschreitenden europäischen Wissensregion geführt hat. Ein inhaltsreicher und raumwirksamer Weg, welchen die Hochschulgeographen von Beginn an mitangestrebt und mitvollendet haben.

Die geschichtlichen Voraussetzungen auf den grundlegenden Gebieten der Erziehung, des theoretischen und praktischen Wissens, des originellen Schul- Berufschul- und technischen Hochschulsystems sowie die Eigenarten des Werdens des rheinischen Einzugsgebiets haben seit den 1950 er Jahren allmählich zu einem wissensgeprägten grenzabgeschwächten internationalvernetzten hochwertigen Raum geführt.

\section{INDEX}

Mots-clés : genèse transfrontalière, nouveau paysage européen du savoir, Rhin supérieur Schlüsselwörter : Bildung eines grenzüberschreitenden Raums, neue europäische Wissensregion, Oberrhein

Keywords : cross-boundary development, new European knowledge landscape, Upper Rhine

\section{AUTEUR}

\section{GABRIEL WACKERMANN}

Professeur émérite à l'Université Paris-Sorbonne - Adresse privée : 180, Route d'Oberhausbergen 67200 Strasbourg - WackermannG@aol.com 